\title{
Focusing Bistatic SAR Data Using the Nonlinear Chirp Scaling Algorithm
}

\author{
Frank H. Wong, Ian G. Cumming, Life Senior Member, IEEE, and Yew Lam Neo, Member, IEEE
}

\begin{abstract}
Bistatic synthetic aperture radar data are more challenging to process than the common monostatic counterparts because the flight geometry is more complicated and the data are usually nonstationary. Whereas time-domain algorithms can handle general bistatic cases, they are very inefficient; therefore, frequency-domain methods are preferred. Several frequencydomain monostatic algorithms have been modified to handle a limited number of bistatic cases, but a general algorithm is sought, which can handle cases such as nonequal platform velocities, nonparallel flight tracks, and high squints. In this paper, we modify the nonlinear chirp scaling (NLCS) algorithm to handle a general case of bistatic data. The key is to use a linear range cell migration correction to reduce the range-azimuth coupling, an NLCS to precondition the data for azimuth compression, and a series expansion to obtain an accurate form of the signal spectrum. The azimuth nonstationarity is handled through the use of invariance regions. Simulations have shown that the modified NLCS algorithm can handle data with more complicated bistatic geometries than the previous algorithms.
\end{abstract}

Index Terms-Bistatic SAR, chirp scaling, perturbation function, point target response, range cell migration (RCM) correction (RCMC), secondary range compression (SRC), synthetic aperture radar (SAR).

\section{INTRODUCTION}

$\mathbf{M}$ ONOSTATIC synthetic aperture radar (SAR) data compensated to a straight line path are azimuth invariant because point targets with the same range of closest approach have the same azimuth phase history. As a result, efficiency can be gained by processing the data in the azimuth frequency domain (FD). Bistatic data, however, are often azimuth variant because both the transmitter and the receiver can assume different motion trajectories. An additional complication is that the range history of a bistatic target is the sum of two hyperbolic range equations, giving a double square-root term in the range equation (called a flat-top hyperbola) [1]. Therefore, the analytical simplicity of the single square root of the monostatic case is no longer available. As a result of these two properties,

Manuscript received June 22, 2007; revised December 18, 2007. This work was supported by the Natural Sciences and Engineering Research Council of Canada. The work of Y. L. Neo was supported by the DSO National Laboratories (Singapore).

F. H. Wong is with MacDonald, Dettwiler and Associates Ltd., Richmond, BC, Canada V6V $2 \mathrm{~J} 3$.

I. G. Cumming is with the Department of Electrical and Computer Engineering, University of British Columbia, Vancouver, BC, Canada V6T 1 Z4.

Y. L. Neo is with the Sensor Division, Radar Techniques Laboratory, DSO National Laboratories, Singapore 118230, Singapore.

Color versions of one or more of the figures in this paper are available online at http://ieeexplore.ieee.org.

Digital Object Identifier 10.1109/TGRS.2008.917599 monostatic SAR processing algorithms must be modified if they are used to handle bistatic data.

Modified $\omega-k$ algorithms were introduced in [2] and [3]. These algorithms work in the 2-D FD and make use of numerical methods to calculate the double square-root phase term. Zhang et al. [4] proposed a wavenumber domain approach based on the concept of an instantaneous Doppler wavenumber to process bistatic SAR data with parallel tracks. Bamler and Boerner [5] and Bamler et al. [6] proposed a focusing algorithm that replaces the analytical SAR transfer functions with numerical equivalents. Their algorithm is able to handle the azimuthinvariant case, including squint. In a separate approach, a preprocessing technique derived from the "Dip Move Out" in the seismic literature [7] is used to transform the bistatic data to a monostatic equivalent [1]. The data can subsequently be focused with any monostatic algorithm. In [8], Monti Guarneiri and Rocca made an extension to [7], in which they reduced a general bistatic configuration to a monostatic configuration. However, such a reduction to monostatic configuration may not be applicable for more extreme bistatic cases. Loffeld et al. [9] derived an approximate bistatic point target spectrum, and several algorithms were developed based on this approach [10], [11]. Natroshvili et al. [12] applied this point target result to focus the azimuth-variant case. The bistatic geometries that can be focused are limited by the accuracy of the point target spectra. Rodríguez-Cassolá et al. [13] made use of the results from [9] to modify the range Doppler algorithm [14] and the chirp scaling algorithm [15] to handle bistatic cases. Most of these modified algorithms are only able to handle azimuthinvariant and mildly bistatic cases.

The original nonlinear chirp scaling (NLCS) algorithm has been shown to be an alternative way to focus monostatic data and has been demonstrated to work on the bistatic configuration, where the transmitter has zero squint and the receiver is stationary [16]. Qiu et al. applied the NLCS algorithm to the case where one of the transmitters or receivers is stationary [17]. This paper further develops the NLCS algorithm to handle bistatic geometries, where the platforms have unequal velocities and their flight paths are not parallel. Recently, an accurate 2-D point target spectrum has been derived by basing on the reversion of a power series for the general bistatic case [18]. It is known as the method of series reversion (MSR). By making use of this point target spectrum result, the NLCS algorithm can be made to handle more general bistatic cases. The algorithm is able to process higher squint and longer wavelength cases than other algorithms. Previously, only inefficient time-domain algorithms, such as back projection [19], have been able to handle general bistatic cases. This paper shows 


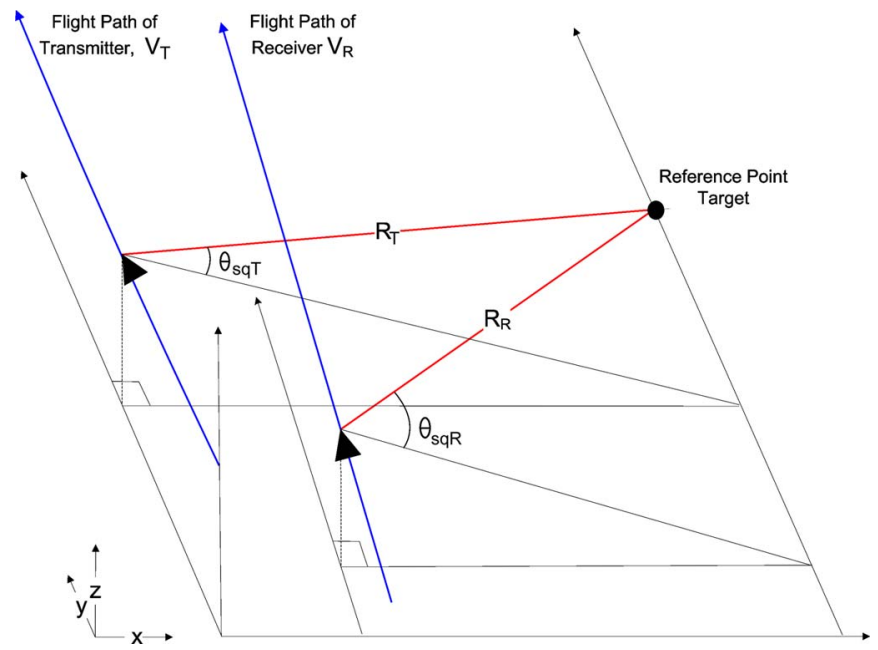

Fig. 1. Aircraft positions and beam geometry at the time when the center of the composite beam crosses the reference target.

how the efficiency of the FD can be obtained with the NLCS algorithm.

This paper begins by introducing a signal model that is suitable for developing the bistatic SAR processing algorithms. Section III explains the principles and limitations of the original NLCS algorithm. Section III-A presents the modified NLCS algorithm without secondary range compression (SRC). The explanation of how SRC can be added to the algorithm to handle wide-aperture cases is left to Appendix A, because it is not important to the understanding of the NLCS algorithm and in the development of the processing equations. Section IV presents the simulation results of a bistatic case, where the platforms have unequal velocities and the flight paths are not parallel.

The focusing limits of the algorithm can be determined by ensuring that the uncompensated range cell migration (RCM) and the final phase errors fall within specified limits. This will ensure that the impulse response has acceptable broadening in resolution and the sidelobe ratios are small enough. The thesis of Neo [20] gives a detailed analysis of how the invariance region can be determined, and a summary is presented in Appendix C.

\section{Bistatic Signal Model}

We consider the general bistatic imaging geometry shown in Fig. 1. Both the transmitter and the receiver are assumed to be flying on straight but nonparallel paths with a constant velocity. The transmitter has a velocity of $V_{T}$, and the receiver has a different velocity $V_{R}$. We use the right-hand Cartesian coordinate system, where the $x-y$ plane is locally tangent to the surface of the Earth. The targets are assumed to lie on this plane, and the velocity vector of the transmitter is parallel to the $y$-axis.

In bistatic operation, one or both of the antennas are steered to obtain an overlapping beam on the ground, and the exposure of a target is governed by this composite beam pattern. The instantaneous range from the transmitter to an arbitrary reference point target is $R_{T}(\eta)$, and the corresponding range from the receiver is $R_{R}(\eta)$. The range time is given by $\tau$, and the azimuth time $\eta$ is chosen to be zero at the composite beam center crossing time (midexposure time) of the reference target. The squint angles $\theta_{\mathrm{sq} T}$ and $\theta_{\mathrm{sq} R}$ shown in Fig. 1 are measured at the composite beam center crossing time of the target.

Assuming that a wide bandwidth encoded signal $p(\tau)$ is upconverted by the transmitter to the carrier frequency $f_{o}$; the transmitted signal can be expressed as

$$
s_{t}(\tau)=\mathcal{R} e\left\{p(\tau) \exp \left(j 2 \pi f_{o} \tau\right)\right\} .
$$

The echo received from a point target experiences a delay that is proportional to the two-way slant range $R(\eta)$

$$
s_{r}(\tau, \eta)=w_{\mathrm{az}}(\eta) s_{t}\left(\tau-\frac{R(\eta)}{c}\right)
$$

where the azimuth envelope $w_{\mathrm{az}}(\eta)$ is given by the composite antenna pattern, and $c$ is the speed of light. The constant amplitude terms in the signal have been omitted. The instantaneous two-way range $R(\eta)$ of the target is given by (3) at the bottom of the page. where $\eta_{\mathrm{bcc}}$ is the beam center crossing time, $R_{T \text { cen }}$ is the range of the transmitter to the point target, and $R_{R \text { cen }}$ is the corresponding range of the receiver, both at $\eta_{\mathrm{bcc}}$ of the target.

After downconversion, the demodulated signal becomes

$$
s(\tau, \eta)=w_{\mathrm{az}}(\eta) p\left(\tau-\frac{R(\eta)}{c}\right) \exp \left\{-j 2 \pi \frac{R(\eta)}{\lambda}\right\}
$$

where $\lambda$ is the radar wavelength.

One way to treat the double hyperbolic range in (3) is to expand it in a power series in time

$$
\begin{aligned}
R(\eta)=R_{\mathrm{cen}}+ & k_{1}\left(\eta-\eta_{\mathrm{bcc}}\right)+k_{2}\left(\eta-\eta_{\mathrm{bcc}}\right)^{2} \\
& +k_{3}\left(\eta-\eta_{\mathrm{bcc}}\right)^{3}+k_{4}\left(\eta-\eta_{\mathrm{bcc}}\right)^{4}+\cdots
\end{aligned}
$$

where $R_{\text {cen }}=R_{T \text { cen }}+R_{R \text { cen }}$ denotes a two-way range at the beam center crossing time. For an arbitrary target, the RCM slope $k_{1}$ can be expressed as

$$
k_{1}=V_{T} \sin \left(\theta_{\mathrm{sq} T}\right)+V_{R} \sin \left(\theta_{\mathrm{sq} R}\right)
$$

$$
\begin{aligned}
R(\eta)= & R_{T}(\eta)+R_{R}(\eta) \\
= & \sqrt{V_{T}^{2}\left(\eta-\eta_{\mathrm{bcc}}\right)^{2}+R_{T \mathrm{cen}}^{2}-2 V_{T}\left(\eta-\eta_{\mathrm{bcc}}\right) R_{T \mathrm{cen}} \sin \left(\theta_{\mathrm{sq} T}\right)} \\
& +\sqrt{V_{R}^{2}\left(\eta-\eta_{\mathrm{bcc}}\right)^{2}+R_{R \mathrm{cen}}^{2}-2 V_{R}\left(\eta-\eta_{\mathrm{bcc}}\right) R_{R \operatorname{cen}} \sin \left(\theta_{\mathrm{sq} R}\right)}
\end{aligned}
$$




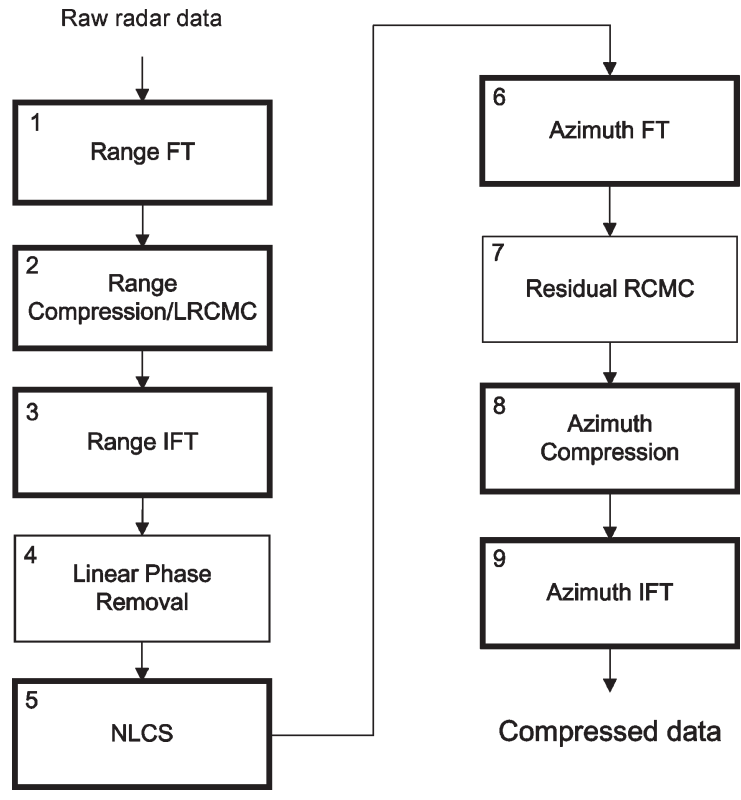

Fig. 2. Functional block diagram of the extended NLCS algorithm (without SRC). The seven thick-lined boxes pertain to the original NLCS algorithm.

and the second derivative coefficient [that governs the azimuth frequency modulation (FM) rate] can be expressed as

$$
k_{2}=\frac{1}{2}\left[\frac{V_{T}^{2} \cos ^{2}\left(\theta_{\mathrm{sq} T}\right)}{R_{T \mathrm{cen}}}+\frac{V_{R}^{2} \cos ^{2}\left(\theta_{\mathrm{sq} R}\right)}{R_{R \mathrm{cen}}}\right] .
$$

The temporal coefficients $k_{1}, k_{2}, \ldots$, are weakly dependent on the range and azimuth of the target (the expressions for the other $k$ coefficients are given in [18]). The $k_{1}$ coefficient is used for the linear phase removal in Section III-C, the $k_{2}$ coefficient is used for the derivation of the perturbation function in Section III-D, and the higher order coefficients are used in defining the azimuth-matched filter in Section III-F, using the MSR.

\section{EXTENDED NLCS ALGORITHM}

The original NLCS algorithm [16] was used to focus the following: 1) monostatic data and 2) bistatic data, where the transmitter is illuminating on broadside and the receiver is stationary. It applied to short-wavelength systems, where the quadratic RCM (QRCM) could be ignored.

The main steps in the original NLCS are shown in the thicklined boxes in Fig. 2. The first three steps implement range compression and linear RCM correction (LRCMC). One result of LRCMC is that the targets with different azimuth FM rates are moved into the same range gate. In order to allow azimuth compression in the FD, NLCS is applied in the time domain to equalize the FM rates along each range cell. This is done by multiplying the signal by a "perturbation function" that has the form of a complex exponential. ${ }^{1}$ Once the azimuth FM rate

\footnotetext{
${ }^{1}$ The term "perturbation" is used because its effect is to alter or perturb the frequency of the signal by a varying amount as the azimuth time progresses. The current form of chirp scaling is called "nonlinear," as the scaling function has a cubic phase term. The original chirp scaling algorithm [15] uses a quadratic phase term, i.e., linear in frequency.
}

is equalized along each range gate, conventional FD azimuth compression can be applied to focus the data. There is some analogy with the method that Moreira et al. used to equalize the FM rates in monostatic SAR processing to allow the use of the spectral analysis algorithm for azimuth compression [21].

\section{A. Algorithm Description}

In [22] and [23], we introduced an extension of the NLCS to focus the bistatic configuration, where the platforms were assumed to have parallel tracks and equal velocities. In [24], we extended the method to nonparallel tracks and nonequal velocities. This paper is an extension of [24], in that it gives a detailed derivation of the processing equations. It also gives a more accurate form of the RCM, using MSR, and it shows how SRC can be incorporated into the NLCS algorithm.

As a key step, we use the MSR to derive the 2-D point target spectrum. By using the analytical form obtained by the MSR, the azimuth-matched filter can be derived in the FD, which allows the efficiency of fast convolution in the azimuth compression operation to be obtained. The resulting accuracy of the spectrum is limited only by the number of terms used in the expansion of (5). When longer apertures and/or higher squints need to be processed, more terms can be kept in the expansion.

Fig. 2 shows the main steps of the extended NLCS algorithm, when SRC is not needed. A discussion of how SRC can be incorporated is given in Appendix A.

Range compression and LRCMC (boxes 1 to 3 ) are done in the range FD. A (box 4) linear phase is applied along the azimuth time variable in the range time domain to remove the Doppler offset that comes from squinted operation. The targets that have been moved to the same range gate now have different FM rates. A (box 5) perturbation function is applied in the azimuth time domain to equalize the FM rates along each range gate. Residual RCMC (box 7) is performed in the range Doppler domain, using a range direction interpolation. This step was ignored in the original NLCS, which concentrated on shortwavelength cases. Accuracy is improved by incorporating this step. Finally, (boxes 8 and 9) azimuth compression is carried out by using an FD matched filter, using invariance regions as needed.

In the subsequent sections, each major stage of the extended NLCS algorithm is analyzed and discussed. An image registration step is usually applied at the end (e.g., to undo LRCMC and register the image to a map datum), but it will not be discussed further.

\section{B. Linear RCM Correction}

The trajectory of the point target contains both linear and nonlinear RCM components in general. The linear component [(6)] arises when the composite antenna beam is squinted. Most of the range Doppler coupling tends to come from the linear component, particularly for short-wavelength systems. This component can be removed with LRCMC, which reduces the need for SRC. Furthermore, LRCMC aligns the trajectories with the azimuth axis to facilitate azimuth compression. 


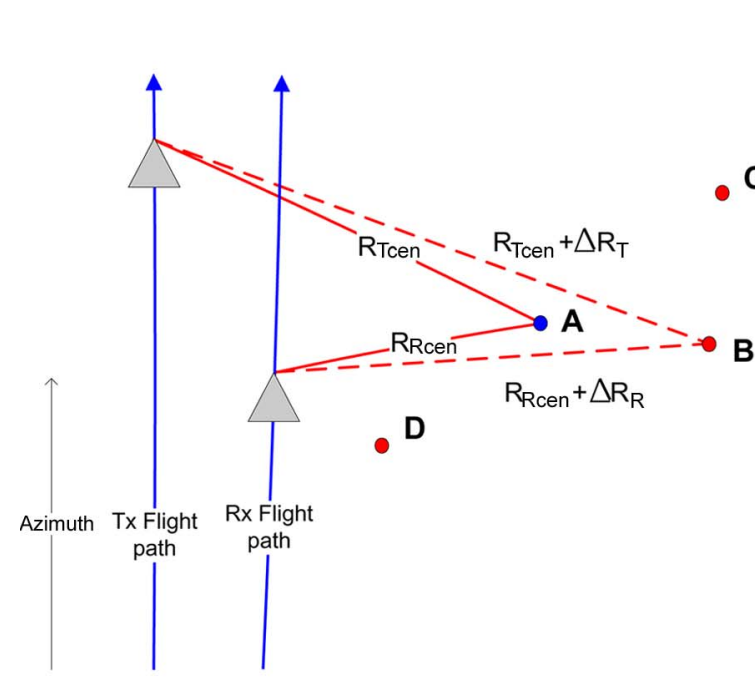

(a)

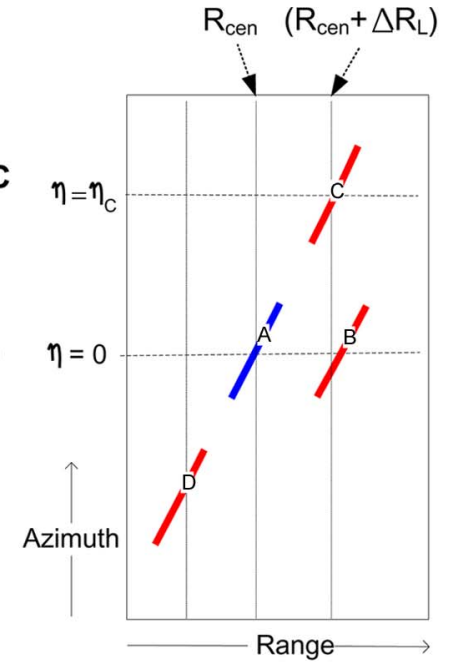

(b)

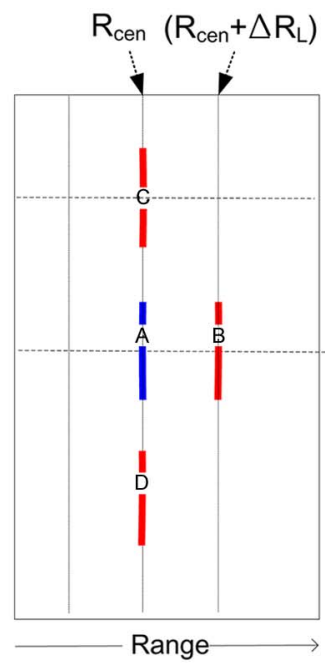

(c)

Fig. 3. Illustration of linear RCMC in the NLCS algorithm. (a) Bistatic SAR imaging geometry. (b) Data before LRCMC. (c) After LRCMC.

In practice, the LRCM slope varies with range because squint angles vary with the target range. In the azimuth-variant bistatic case, the slope varies with the azimuth as well. A practical way to deal with this problem is to perform LRCMC within an invariance region to keep the variations of squint angles small. The amount of (two-way) range shift is computed from a reference point, for example, $\eta=0$, as

$$
\delta_{r}(\eta)=k_{1} \eta=\left[V_{T} \sin \left(\theta_{\mathrm{sq} T}\right)+V_{R} \sin \left(\theta_{\mathrm{sq} R}\right)\right] \eta .
$$

The LRCMC interpolation is usually combined with the range compression in the range $\mathrm{FD}$, using a phase ramp.

To illustrate the LRCMC processing of the NLCS algorithm, consider a simple bistatic flight geometry, such as the one shown in Fig. 3(a), with four point targets. Target B has the same minimum slant range as target $\mathrm{C}$ and the same beam center crossing time as target A, as shown in Fig. 3(b). Targets A, $\mathrm{C}$, and D have a similar LRCM slope (at an angle given by the composite squint angle) and lie in the same range cell after LRCMC, as shown in Fig. 3(c). For processing purposes, target $\mathrm{A}$ is chosen as the reference point (nominally at midswath), and the beam center crossing time of this target is set to zero for convenience. Letting $\eta_{C}=\eta_{\mathrm{bcc}}$ for target $\mathrm{C}$, we can write

$$
R_{\mathrm{cen} C}=R_{\mathrm{cen} A}+k_{A 1} \eta_{C}=R_{\operatorname{cen} A}+\Delta R_{L}
$$

where the $R$ terms are interpreted as the two-way slant range of the respective targets at the composite beam center crossing time. After LRCMC, targets A, C, and D lie in the same range cell, as shown in Fig. 3(c).

In practice, the targets after LRCMC have some higher order residual RCMs, which may or may not be significant. For example, the instantaneous slant range of target $\mathrm{A}$ after LRCMC is given by

$$
R_{\operatorname{lrcm} A}(\eta)=R_{\operatorname{cen} A}+k_{A 2} \eta^{2}+k_{A 3} \eta^{3}+k_{A 4} \eta^{4}+\cdots
$$

where the subscripts of the $k$ terms indicate that they are the coefficients $k_{i}$ in (5) for target A.

\section{Linear Phase Removal}

Although LRCMC removes the linear translation component of the target's trajectory, it does not remove the Doppler shift that is also caused by the squint. The Doppler shift is a linear phase term in azimuth time, which should be removed to facilitate the application of the MSR [25]. The removal of the linear phase term can be done in the azimuth time domain by multiplication with the phase function

$$
\begin{aligned}
s_{\operatorname{lrcm}}(\eta) & =\exp \left\{j \frac{2 \pi}{\lambda} k_{A 1} \eta\right\} \\
& =\exp \left\{j \frac{2 \pi}{\lambda}\left(V_{T} \sin \left(\theta_{\mathrm{sq} T}\right)+V_{R} \sin \left(\theta_{\mathrm{sq} R}\right)\right) \eta\right\}
\end{aligned}
$$

where the squint angles (and the velocities in the satellite case) are mildly range dependent.

The signal from the reference target A after LRCMC and linear phase removal is given by

$$
s_{A}(\tau, \eta) \approx \rho_{r}\left\{\tau-\frac{R_{\operatorname{lrcmA}}(\eta)}{c}\right\} w_{\mathrm{az}}(\eta) \exp \left\{-j 2 \pi \frac{R_{\operatorname{lrcm} A}(\eta)}{\lambda}\right\}
$$

where $\rho_{r}(\cdot)$ is the sinc-like range-compressed pulse envelope. In similar, the signal for target $C$ after LRCMC and linear phase removal is given by

$$
\begin{array}{r}
s_{C}(\tau, \eta) \approx \rho_{r}\left\{\tau-\frac{R_{\operatorname{lrcm} C}(\eta)}{c}\right\} w_{\mathrm{az}}\left(\eta-\eta_{C}\right) \\
\times \exp \left\{-j 2 \pi \frac{R_{\operatorname{lrcm} C}(\eta)}{\lambda}\right\}
\end{array}
$$

where

$$
\begin{aligned}
R_{\operatorname{lrcm} C}(\eta)= & R_{\operatorname{cen} A}+k_{C 2}\left(\eta-\eta_{C}\right)^{2} \\
& +k_{C 3}\left(\eta-\eta_{C}\right)^{3}+k_{C 4}\left(\eta-\eta_{C}\right)^{4}+\cdots .
\end{aligned}
$$

These equations express the different FM rates of targets A and $\mathrm{C}$, as $k_{C 2} \neq k_{A 2}$. This information is used in the next 
(a)

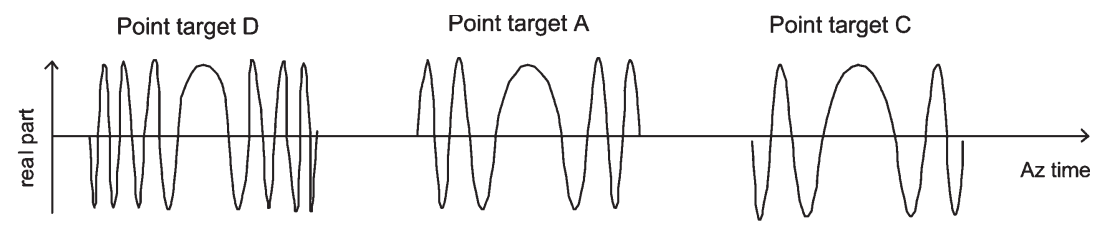

(b)

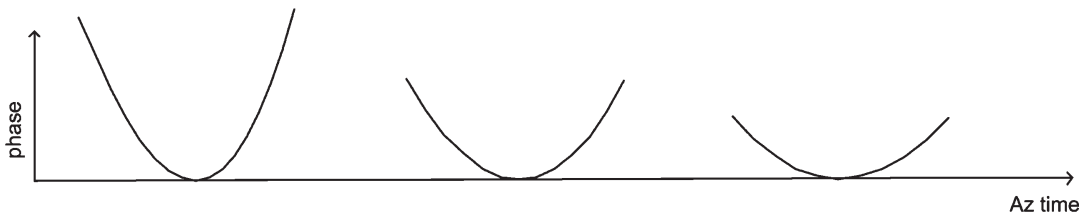

(c)

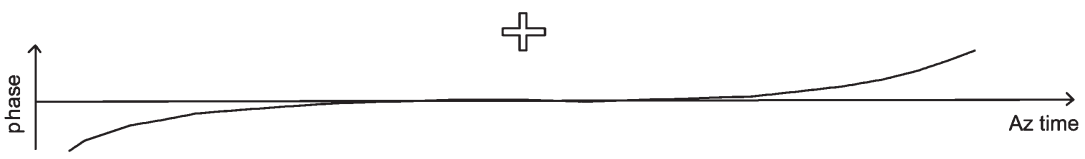

(d)

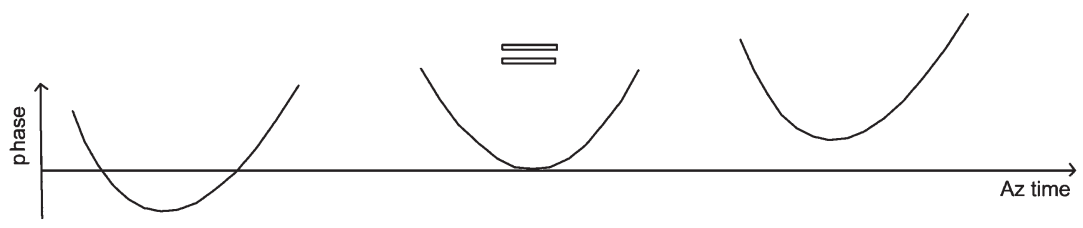

(e)

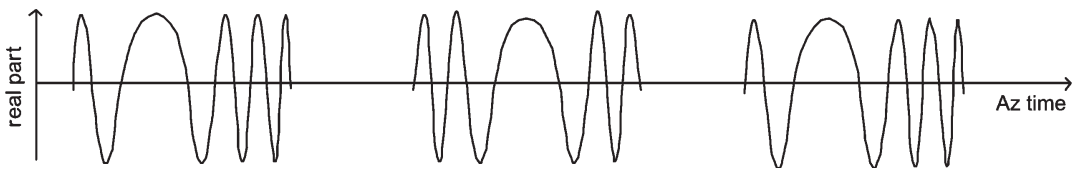

Fig. 4. Illustration of the effects of perturbation in parallel case.

section to derive the perturbation function that equalizes the azimuth FM rates of these targets.

\section{FM Rate Equalization Using NLCS}

The azimuth signal in a given range cell now consists of a group of point targets that originated from different ranges and, consequently, have different azimuth FM rates. Therefore, it would not be accurate to compress the whole azimuth array using a single FD matched filter. To tackle this problem, the NLCS algorithm equalizes the dominant quadratic phase term of each target, using a perturbation function [16]. Because the higher order phase terms do not change as rapidly with range and azimuth as the quadratic term does, they can be assumed to be constant in the processed region for the purposes of azimuth compression. This process can be viewed as a type of preprocessing, because it effectively makes the dominant secondorder phase component azimuth invariant before applying the azimuth compression filter.

Fig. 4 shows the principle of equalizing the FM rate using the perturbation function. In order to simplify the illustration, the Doppler offset due to squint is not shown. Only the quadratic part of the phase is shown, which is sufficient to derive the perturbation function. Later, when we derive the azimuthmatched filter in Section III-F, the higher order terms in the range equation will be used, as they are needed for accuracy at that stage.

We consider targets $\mathrm{A}, \mathrm{C}$, and $\mathrm{D}$, which lie in the same range cell after LRCMC, as shown in Fig. 3. Fig. 4(a) shows the real part of the azimuth FM signal of the three point targets, and Fig. 4(b) shows the corresponding phase of each azimuth signal. The three phase curves have different FM rates (second derivatives) because this parameter varies with the original range position of the target.

The perturbation function is given a cubic form to equalize the FM rates of the targets. An appropriate cubic function is shown in Fig. 4(c). This cubic phase is added to the phase in (b) along azimuth time to obtain the three target phases shown in Fig. 4(d). We can see how the phase of each target has been altered by the perturbation to achieve the same FM rate. However, a phase offset has been introduced for each target, which can be removed later. Finally, Fig. 4(e) shows the real part of the perturbed signal of the three point targets, where a small Doppler shift can be seen.

To determine the cubic phase term of the perturbation function, the phases of targets $\mathrm{A}$ and $\mathrm{C}$ after perturbation are written down. Subsequently, the cubic coefficient of the perturbation function is selected in such a way that the FM rates of targets A and $\mathrm{C}$ become equalized.

The azimuth signal for reference target $\mathrm{A}$, after introducing the cubic perturbation function $\exp \left\{j \pi \alpha \eta^{3}\right\}$, is given by

$$
s_{A \text { pert }}(\tau, \eta)=s_{A}(\tau, \eta) \exp \left\{j \pi \alpha \eta^{3}\right\}
$$

where the parameter $\alpha$ is the coefficient of the cubic perturbation function. Its expression is derived in Appendix B and given by

$$
\begin{aligned}
\alpha=\frac{1}{3}\left[\frac{V_{T}^{2} \cos ^{2}\left(\theta_{\mathrm{sq} T}\right)}{\lambda R_{T \operatorname{cen} A} R_{\operatorname{cen} A}}+\frac{V_{R}^{2} \cos ^{2}\left(\theta_{\mathrm{sq} R}\right)}{\lambda R_{R \operatorname{cen} A} R_{\operatorname{cen} A}}\right] \\
\times\left(V_{T} \sin \left(\theta_{\mathrm{sq} T}\right)+V_{R} \sin \left(\theta_{\mathrm{sq} R}\right)\right) .
\end{aligned}
$$


The resulting perturbed signal is also derived in Appendix B, and it is given by

$$
\begin{aligned}
s_{C \text { pert }}^{\prime} & \left(\tau, \eta_{1}\right) \\
\approx & \rho_{r}\left(\tau-\frac{R_{\mathrm{cen} A}}{c}\right) w_{\mathrm{az}}\left(\eta_{1}\right) \\
& \times \exp \left\{j \pi \alpha \eta_{1}^{3}\right\} \exp \left\{j \pi \alpha 3 \eta_{C}^{2} \eta_{1}\right\} \exp \left\{j \pi \alpha \eta_{C}^{3}\right\} \\
& \times \exp \left\{-j \frac{\pi}{\lambda}\left(\frac{V_{T}^{2} \cos ^{2}\left(\theta_{\mathrm{sq} T}\right)}{R_{T \operatorname{cen} A}}+\frac{V_{R}^{2} \cos ^{2}\left(\theta_{\mathrm{sq} R}\right)}{R_{R \operatorname{cen} A}}\right) \eta_{1}^{2}\right\}
\end{aligned}
$$

so that the phase modulation is now independent of target position.

The four exponential terms in (17) can be interpreted as follows.

1) The first term is a cubic phase modulation, which is the same for all targets. This cubic phase is caused by the perturbation process.

2) The second phase term is a small Doppler shift. For example, the Doppler shift for target $\mathrm{C}$ is as follows:

$$
f_{\text {shift }}=\frac{1}{2 \pi}\left[\frac{d\left(3 \pi \alpha \eta_{C}^{2} \eta_{1}\right)}{d \eta_{1}}\right]=\frac{3 \alpha \eta_{C}^{2}}{2} .
$$

The amount of spectrum shift is proportional to the square of $\eta_{C}$ (the azimuth time offset from $\eta=0$ ). The point targets that are furthest away from the reference experience the most spectrum shift. All point targets are shifted in the same direction, as can be seen in Fig. 4(e). This Doppler shift generally does not present a problem because the azimuth signal is usually oversampled by $20 \%$. As long as the shift in the spectrum band stays within this constraint, no aliasing of the spectrum will occur (refer to a numerical example in Section IV-A, where the shift is only $6 \%$ of the azimuth bandwidth).

3) The third phase term is a constant, which depends on the position of the target. This constant phase term has no effect on the focusing process. It can be ignored if a magnitude image is the final product. This term raises or lowers the point target phase, as shown in Fig. 4(d).

4) The fourth exponential term is the linear FM modulation, which is now the same for all targets. This is the soughtafter result of the perturbation operation.

To summarize, the final azimuth phase modulation is contained in the first and fourth exponential terms in (17), and this modulation is the same for all targets in the same range gate.

As a simple check on the perturbation coefficient, the parameters are set for the limiting case of a monostatic geometry. The velocity is set to $V_{r}=V_{T}=V_{R}$, the squint angle is set to $\theta_{\mathrm{sq}}=\theta_{\mathrm{sq} T}=\theta_{\mathrm{sq} R}$, and the one-way range becomes $R_{s}=R_{\text {cen } A} / 2=R_{\text {cen } T}=R_{\text {cen } R}$. Substituting these values into (16), the perturbation coefficient becomes

$$
\alpha_{m}=\frac{2}{3}\left(\frac{V_{r}^{3} \cos ^{2}\left(\theta_{\mathrm{sq}}\right) \sin \left(\theta_{\mathrm{sq}}\right)}{\lambda R_{s}^{2}}\right)
$$

which is the same as the perturbation coefficient derived in [16] for the monostatic configuration.

\section{E. Residual RCMC}

After LRCMC, the RCM includes quadratic and higher order terms, although the higher order terms are usually very small compared to the quadratic term. Uncorrected range curvature leads to impulse response degradation in both the range and azimuth directions. The residual RCM is usually kept at less than half a range resolution cell, so that range broadening is negligible.

Residual RCMC is carried out by using a range direction interpolator in the range Doppler domain. Applying an azimuth Fourier transform (FT) to the signal in (12), the range Doppler signal of target A can be written as

$\begin{aligned} S_{A}\left(\tau, f_{\eta}\right)=\rho_{r}\left(\tau-\frac{1}{c}\right. & \left.\left(R_{\mathrm{cen} A}+R_{\mathrm{curv}}\left(f_{\eta}\right)\right)\right) \\ & \times w_{\mathrm{az}}\left(f_{\eta}\right) \exp \left\{-j \phi_{\mathrm{az} A}\left(f_{\eta}\right)\right\}\end{aligned}$

where $R_{\text {curv }}\left(f_{\eta}\right)$ describes the range curvature of the point target in the range Doppler domain, and $\phi_{\mathrm{az} A}($.$) is the azimuth$ phase modulation. An expression for the azimuth phase term $\phi_{\mathrm{az} A}($.$) is derived in Section III-F.$

Using the analytical MSR spectrum result in [18], the range curvature $R_{\text {curv }}\left(f_{\eta}\right)$ can be derived with relative ease [25]

$R_{\text {curv }}\left(f_{\eta}\right)=\frac{\lambda^{2}}{4 k_{2}} f_{\eta}^{2}+\lambda^{3}\left[\frac{k_{3}}{4 k_{2}^{3}}\right] f_{\eta}^{3}+3 \lambda^{4}\left[\frac{9 k_{3}^{2}-4 k_{2} k_{4}}{64 k_{2}^{5}}\right] f_{\eta}^{4}+\cdots$

The range curvature does not have a strong dependence on range. As an example, consider a 1-m resolution L-band system with a transmitter range of $13.4 \mathrm{~km}$ and a receiver range of $13.9 \mathrm{~km}$. The transmitter/receiver separation or baseline is $5 \mathrm{~km}, \theta_{\mathrm{sq} T}=30^{\circ}, \theta_{\mathrm{sq} R}=45.3^{\circ}$, and the range swath width is $5 \mathrm{~km}$. It is found that the residual RCM is quite large at ten range resolution cells. However, the residual RCM change between the midswath and edge targets (measured at the end of the azimuth aperture) is only $10 \%$ of a resolution cell. Thus, the residual $\mathrm{RCM}$ of targets $\mathrm{A}$ and $\mathrm{C}$ coincides quite closely in the range Doppler domain; thus, it can be removed with the same RCMC shift.

Trajectories become parallel to the azimuth frequency axis after the residual RCMC operation. For efficient implementation of the NLCS algorithm, range curvature correction is normally done after the FM rate equalization stage. This is because FM rate equalization is performed in the azimuth time domain, whereas residual RCMC and azimuth compression are both done in the range Doppler domain.

\section{F. Azimuth Compression}

The derivation of the coefficient $\alpha$ has ignored all phase terms in the signal higher than the quadratic, as shown in (32) in Appendix B. As explained in the appendix, the perturbed signal shows that only the linear FM rate of all targets is equalized by the NLCS. Whereas the terms higher than the quadratic are not equalized over azimuth, these terms should be taken into account in the azimuth-matched filter, which are set for the reference target A. For other targets, there is a residual phase error after azimuth compression due to the difference between 
these higher order phase terms and those of target A. Usually, these cubic and higher order phase errors are not significant compared to the others discussed in Appendix C.

The higher order terms for target A can be incorporated in the azimuth FD matched filter by performing an FT on (15), using (12), while keeping the terms up to the fourth order in (10), as

$$
\begin{array}{r}
S_{\mathrm{az} A}\left(\tau, f_{\eta}\right) \approx \int \rho_{r}\left(\tau-\frac{R_{\mathrm{cen} A}}{c}\right) w_{\mathrm{az}}(\eta) \exp \left\{-j 2 \pi f_{\eta} \eta\right\} \\
\quad \exp \left\{-j \frac{2 \pi}{\lambda}\left(k_{A 2} \eta^{2}+k_{A 3} \eta^{3}+k_{A 4} \eta^{4}\right)+j \pi \alpha \eta^{3}\right\} d \eta .
\end{array}
$$

Note that the constant phase term $\exp \left\{-j(2 \pi / \lambda) R_{\operatorname{cen} A}\right\}$ has been ignored here and in subsequent equations. Continuing with the formulation, using the point target spectrum result from MSR and the principle of stationary phase [25], the azimuth frequency $f_{\eta}$ and the azimuth time $\eta$ are now related by

$$
\begin{aligned}
& f_{\eta}(\eta)=-\frac{1}{\lambda}\left(2 k_{A 2} \eta+3 k_{A 3} \eta^{2}+4 k_{A 4} \eta^{3}\right)+\frac{3}{2} \alpha \eta^{2} \\
& \eta\left(f_{\eta}\right)=A_{1} f_{\eta}+A_{2} f_{\eta}^{2}+A_{3} f_{\eta}^{3}+\cdots
\end{aligned}
$$

where

$$
\begin{aligned}
& a_{1}=-\frac{2}{\lambda} k_{A 2} \quad a_{2}=\left(\frac{3}{2} \alpha-\frac{3}{\lambda} k_{A 3}\right) \quad a_{3}=-\frac{4}{\lambda} k_{A 4} \\
& A_{1}=\frac{1}{a_{1}} \quad A_{2}=-\frac{a_{2}}{a_{1}^{3}} \quad A_{3}=\frac{2 a_{2}^{2}-a_{1} a_{3}}{a_{1}^{5}} .
\end{aligned}
$$

The FD matched filter is the conjugate of $S_{\mathrm{az} A}$, and it is given by

$$
h_{\mathrm{amf}}\left(f_{\eta}\right)=\exp \left\{-j \phi_{\mathrm{amf}}\left(\eta\left(f_{\eta}\right)\right)\right\}
$$

where

$\phi_{\mathrm{amf}}\left(f_{\eta}\right)=-\frac{2 \pi}{\lambda}\left(k_{A 2} \eta^{2}+k_{A 3} \eta^{3}+k_{A 4} \eta^{4}\right)+\pi \alpha \eta^{3}-2 \pi f_{\eta} \eta$

and all $\eta$ 's on the right-hand side are functions of $f_{\eta}$, as given in (24).

The matched filter in the azimuth FD can now be computed numerically for each range cell (the $k$ 's depend on range). For each value of $f_{\eta}$, the time at the stationary point of the integrand in (22) is found in (24) and substituted into (27).

Misregistration: The azimuth-matched filter is evaluated by using the reference point target signal given in (32). It focuses all the point targets in the azimuth array to their respective stationary points. Ideally, target $\mathrm{C}$ should be registered to $\eta_{1}=0$. As can be seen in Fig. 4(d) and (e), the edge targets (targets C and $\mathrm{D}$ ) will be misregistered to the right.

To find the misregistration, the position of the stationary point can be solved by setting the differential of phase (28) to zero and finding its roots. Using target $\mathrm{C}$ as an example again

$$
\begin{aligned}
\frac{d \phi_{\mathrm{az} C}}{d \eta_{1}}\left(\tau, \eta_{1}\right)= & -\frac{2 \pi}{\lambda}\left(\frac{V_{T}^{2} \cos ^{2}\left(\theta_{\mathrm{sq} T}\right)}{R_{T \operatorname{cen} A}}+\frac{V_{R}^{2} \cos ^{2}\left(\theta_{\mathrm{sq} R}\right)}{R_{R \operatorname{cen} A}}\right) \eta_{1} \\
& +3 \pi \alpha\left(\eta_{1}^{2}+\eta_{C}^{2}\right) \\
= & 0 .
\end{aligned}
$$
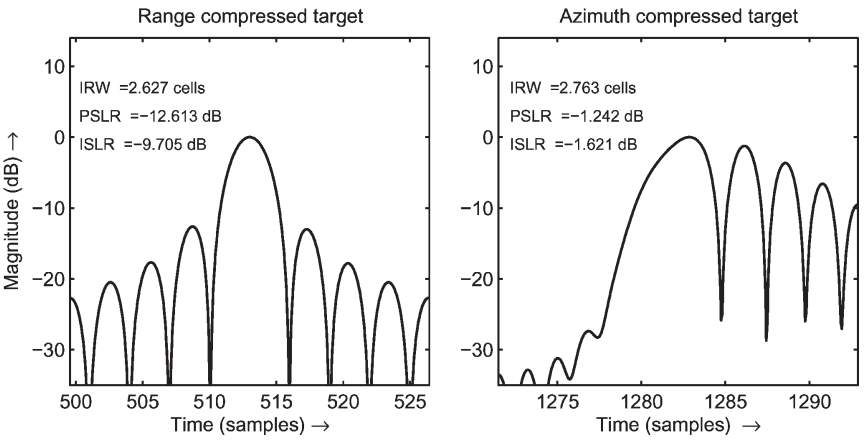

Fig. 5. Impulse response using an azimuth-matched filter with second-order phase.
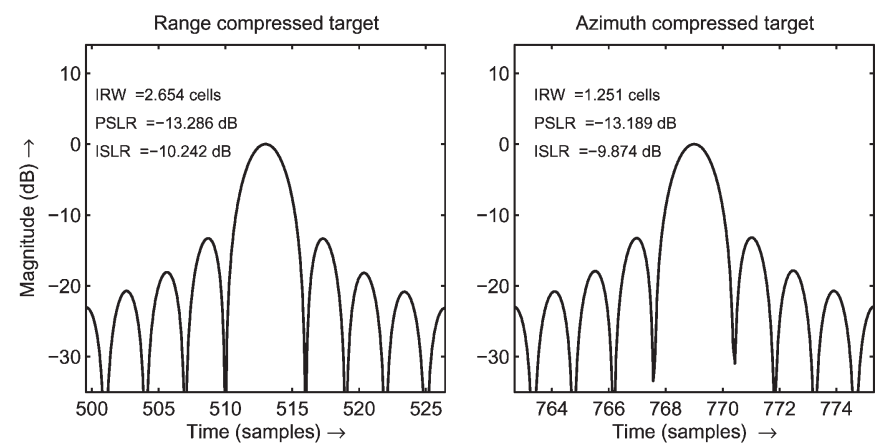

Fig. 6. Impulse response improvement when the cubic phase term is added to the azimuth-matched filter.

The amount of misregistration varies in a parabolic fashion with the azimuth. The misregistration is usually small, and it can be ignored in most cases. It can also be removed during the image registration stage. In practice, it is sufficient to use a few points to fit a second-degree polynomial to this misregistration equation and interpolate the image for correct registration.

Discussion: The importance of adding the cubic phase term to the matched filter can be illustrated with a simple C-band monostatic case with a range and azimuth resolution of $1 \mathrm{~m}$ and a squint angle of $30^{\circ}$. The slant range is $14 \mathrm{~km}$. The ideal impulse response width is 2.65 cells in range and 1.25 cells in azimuth. Rectangular weighting has been used in the processing, and the ideal peak sidelobe ratio (PSLR) and integrated sidelobe ratio (ISLR) are -13.3 and $-10.0 \mathrm{~dB}$, respectively.

Using only the quadratic term [the last exponential term in (17)] in the azimuth-matched filter, the azimuth impulse response is poor, as shown in Fig. 5. The azimuth broadening is more than $100 \%$, and the ISLR is bad. With the inclusion of the cubic phase term in (22), the impulse response is improved significantly, as shown in Fig. 6.

\section{Simulation Example}

To verify the ability of the NLCS to focus the data collected on nonparallel tracks with unequal velocities, a simulation using airborne SAR parameters given in Table I is performed. An appreciable amount of squint is assumed for both the transmitter and receiver antennas, but not enough to require SRC. A residual QRCM of 1.9 range resolution cells is present; therefore, residual RCMC is necessary.

The simulation uses an array of 25 targets, which are laid out on a $1.6-\mathrm{km}^{2}$ grid in ground range/azimuth, as shown 
TABLE I

Simulation PARAMETERS FOR A SLIGHTLY NONPARALLEL FLIGHT GEOMETRY

\begin{tabular}{|c|c|c|}
\hline Simulation parameters & Transmitter & Receiver \\
\hline \hline Velocity in x-direction & $0 \mathrm{~m} / \mathrm{sec}$ & $20 \mathrm{~m} / \mathrm{sec}$ \\
\hline Velocity in y-direction & $200 \mathrm{~m} / \mathrm{sec}$ & $220 \mathrm{~m} / \mathrm{sec}$ \\
\hline Velocity in z-direction & $0 \mathrm{~m} / \mathrm{sec}$ & $0 \mathrm{~m} / \mathrm{sec}$ \\
\hline Radar wavelength & \multicolumn{2}{|c|}{$0.0566 \mathrm{~m}$} \\
\hline Range bandwidth & \multicolumn{2}{|c|}{$100 \mathrm{MHz}$} \\
\hline Doppler bandwidth & \multicolumn{2}{|c|}{$105 \mathrm{~Hz}$} \\
\hline Integration time & \multicolumn{2}{|c|}{$5.2^{\circ}$} \\
\hline Angle between tracks & $3000 \mathrm{~m}$ & $2000 \mathrm{~m}$ \\
\hline Aircraft altitude & $16533 \mathrm{~m}$ & $13498 \mathrm{~m}$ \\
\hline One-way range to target at $\eta=0$ & $30.0^{\circ}$ & $47.3^{\circ}$ \\
\hline Squint angle at $\eta=0$ & \multicolumn{2}{|c|}{$4392 \mathrm{~m}$} \\
\hline Distance between airplanes at $\eta=0$ & \multicolumn{2}{|c|}{$4243 \mathrm{~m}$} \\
\hline Minimum distance between airplanes & \multicolumn{2}{|c|}{$4554 \mathrm{~m}$} \\
\hline Maximum distance between airplanes & \multicolumn{2}{|c|}{$0.179 \mathrm{sec}^{-3}$} \\
\hline Nominal value of $\alpha$ &
\end{tabular}

in Fig. 7. This point target layout corresponds to the range invariance region that limits the resolution broadening to $3 \%$, as determined by the analysis in Appendix C (some applications may be able to allow more broadening). A rectangular window is used in both the range and azimuth processing, i.e., no weighting is used. The oversampling ratio is 2.0 in range and 2.66 in azimuth; thus, the theoretical range resolution is $1.35 \mathrm{~m}$ (1.76 range cells), and the azimuth resolution is $1.6 \mathrm{~m}$ (2.36 azimuth cells), which are based on the definitions of resolution given in [26] and [27].

\section{A. Simulation Results}

The simulated data were processed by using the extended NLCS algorithm in Section III-A, and the resulting image is shown in Fig. 8. The reference point target is well focused and has negligible broadening in both range and azimuth. Its ISLR and PSLR values are within $0.05 \mathrm{~dB}$ of the theoretical values. The other targets are well focused in range- the range PSLR deviates from the theoretical value of $-13.3 \mathrm{~dB}$ by less than $0.1 \mathrm{~dB}$, and the range ISLR is within $0.1 \mathrm{~dB}$ from the expected value of $-10.0 \mathrm{~dB}$.

Targets 5 and 21 are the furthest from the scene center and therefore suffer the largest azimuth phase degradation. The impulse response of these two point targets are shown in Figs. 9 and 10, respectively. For these edge targets, there is negligible broadening in range, whereas azimuth broadening is less than $2.5 \%$. The ISLR degradation is less than $1.5 \mathrm{~dB}$, and the PSLR has a degradation of less than $2 \mathrm{~dB}$ from the theoretical value. The shift in the azimuth spectrum is $17.2 \mathrm{~Hz}$, which is about $6 \%$ of the bandwidth, and hence, no aliasing occurs.

The size of the invariance region is determined by analyzing the allowable residual RCM and phase error and their impact on the impulse response, as in Appendix C. It has been found that the invariance region has been limited to a ground range of
1.6 and $1.84 \mathrm{~km}$ in the azimuth direction for this example, using $3 \%$ as a resolution broadening criterion.

\section{B. Discussion}

In a subsequent operation, the image is geometrically rectified to the ground plane, using interpolators. The result is shown in Fig. 11, where the registered targets are seen to lie on a square grid. The misregistration [(28)] caused by the chirp scaling was up to $7 \mathrm{~m}$ - this was corrected during the registration operation.

The theory and simulation in this paper have not considered the phase preservation of the processing algorithm. Various operations, such as the linear phase removal and the NLCS, affect the image phase. If necessary, the correct phase can be reinstated at the end of the processing, as was done in the original chirp scaling paper [15].

\section{SUMMARY}

The NLCS algorithm discussed in this paper is an extension of the original NLCS algorithm. The algorithm is extended to focus the bistatic configurations, where the platforms assume nonparallel motion with unequal velocities. The formulation of the NLCS algorithm is aided by the analytical spectrum obtained by using the MSR. The more accurate spectrum so obtained allows the extended NLCS algorithm to handle longer wavelength and higher squint cases.

The processing efficiency of NLCS is improved by performing azimuth compression in the FD. In many cases, SRC is not needed. However, if the residual RCMC is large after LRCMC, SRC can be added to the algorithm at the expense of additional complexity.

A simulation was used to verify the effectiveness of the NLCS algorithm. Nonparallel paths, nonequal velocities, and squint were simulated, and the point targets were found to be well focused.

Operations such as linear phase removal and chirp scaling were shown to introduce phase changes, Doppler shifts, and target misregistration. These changes are relatively small, and they can be compensated at the end if required.

\section{APPENDIX A}

\section{EXTENDED NLCS ALGORITHM-2 (WITH SRC)}

This appendix shows how the NLCS processing is modified when SRC is needed.

Most of the range-azimuth coupling is eliminated by the removal of the linear component of the RCM. However, for very wide apertures, e.g., L-band and 1-m resolution, the remaining coupling can still be significant. The remaining coupling is caused by the residual range curvature, which can be as large as tens of range cells. For these cases, SRC is necessary before moving on to range curvature correction.

SRC can be added to the NLCS algorithm by using the steps outlined in Fig. 12. A range IFT is needed in step 3 so that the linear phase removal can be applied in the time domain. Range and azimuth FTs are performed so that SRC can be applied with the correct dependencies of the 2-D FD. A range IFT is then performed to transform the signal to the 


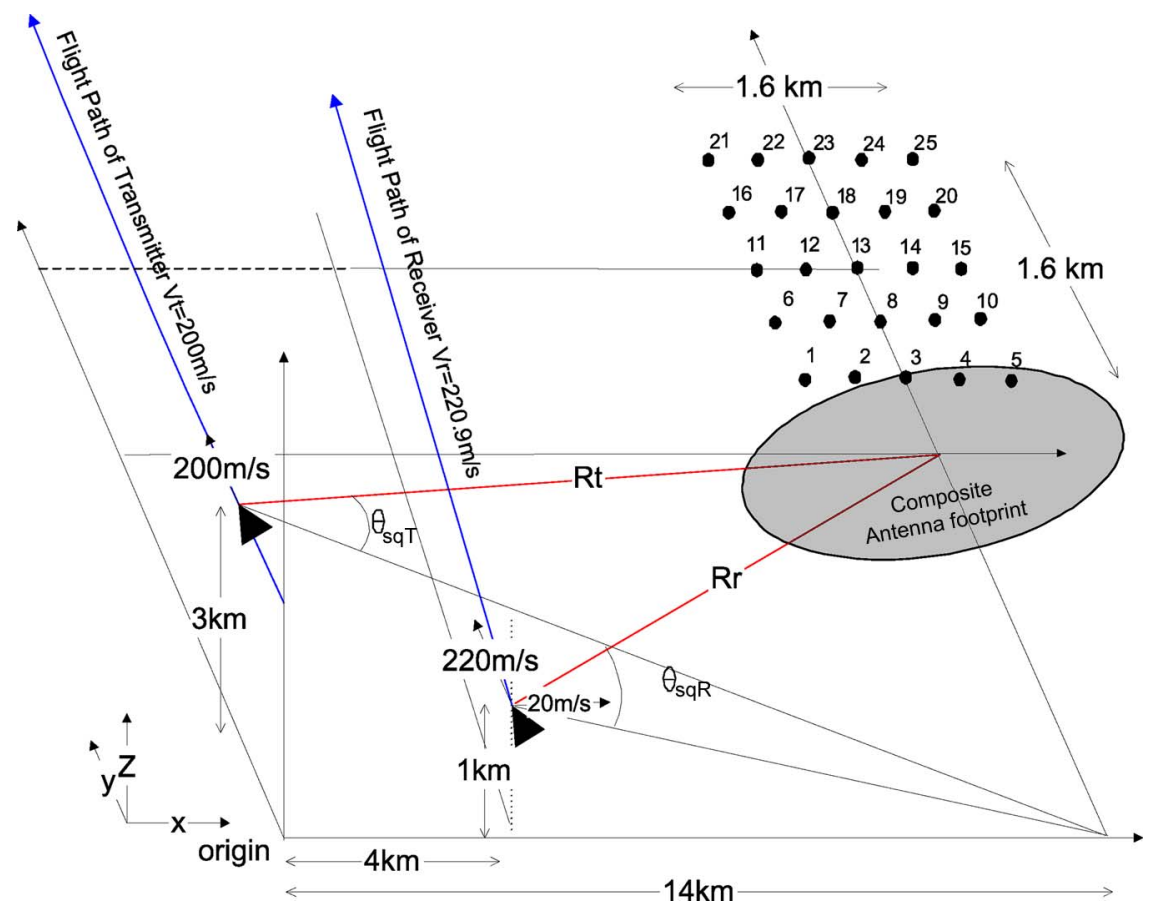

Fig. 7. Flight geometry and target layout for the simulation. The flight paths are $5^{\circ}$ nonparallel.

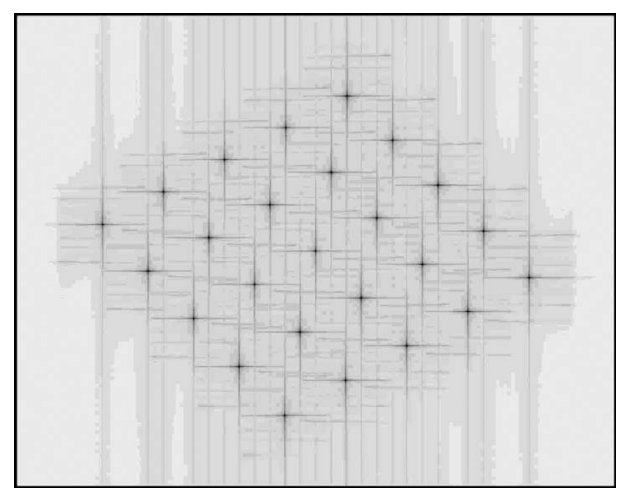

Fig. 8. Twenty-five simulated point targets, which are processed by using the NLCS algorithm, without SRC.
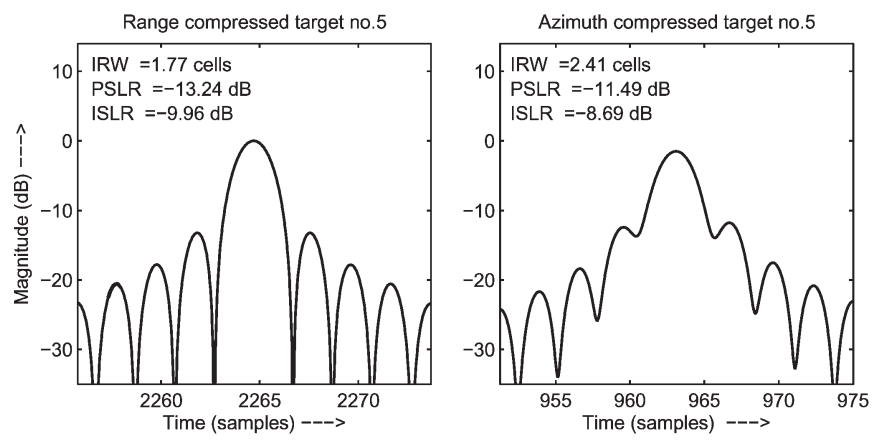

Fig. 9. Impulse response of corner target 5.

range Doppler domain, so that residual RCMC can be applied in this domain. An azimuth IFT is performed; thus, the NLCS FM chirp equalization can be applied in the time domain. RCMC is performed before NLCS because range curvature can spread over many range cells. Finally, azimuth compression is processed in the range Doppler domain to focus the image.
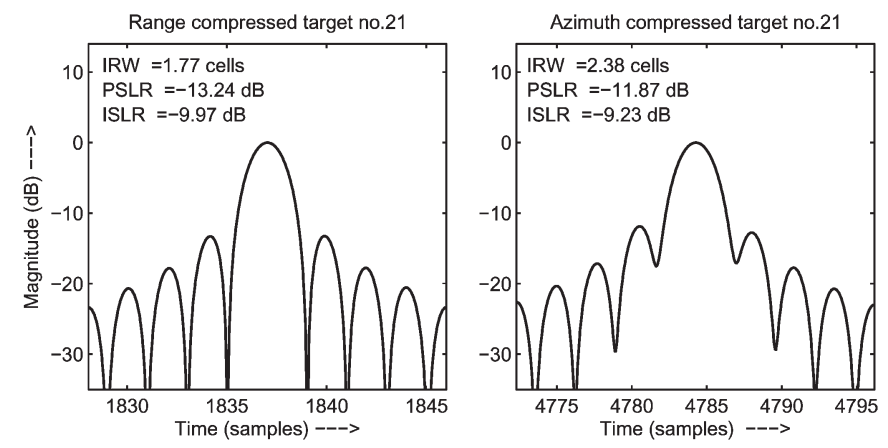

Fig. 10. Impulse response of corner target 21.

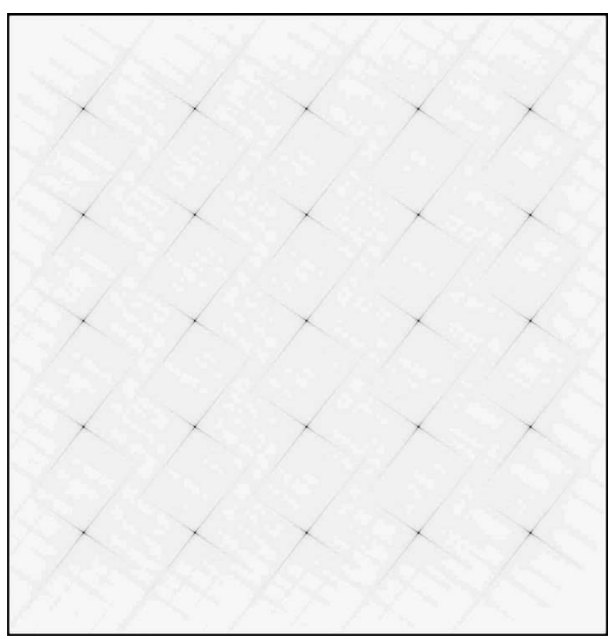

Fig. 11. Image of Fig. 8 registered to the ground plane.

The SRC is evaluated at a reference range. It is applied in the 2-D FD because its main dependence is on range and azimuth frequencies. The phase of the SRC function can be derived by using the analytical point target result obtained by the MSR 


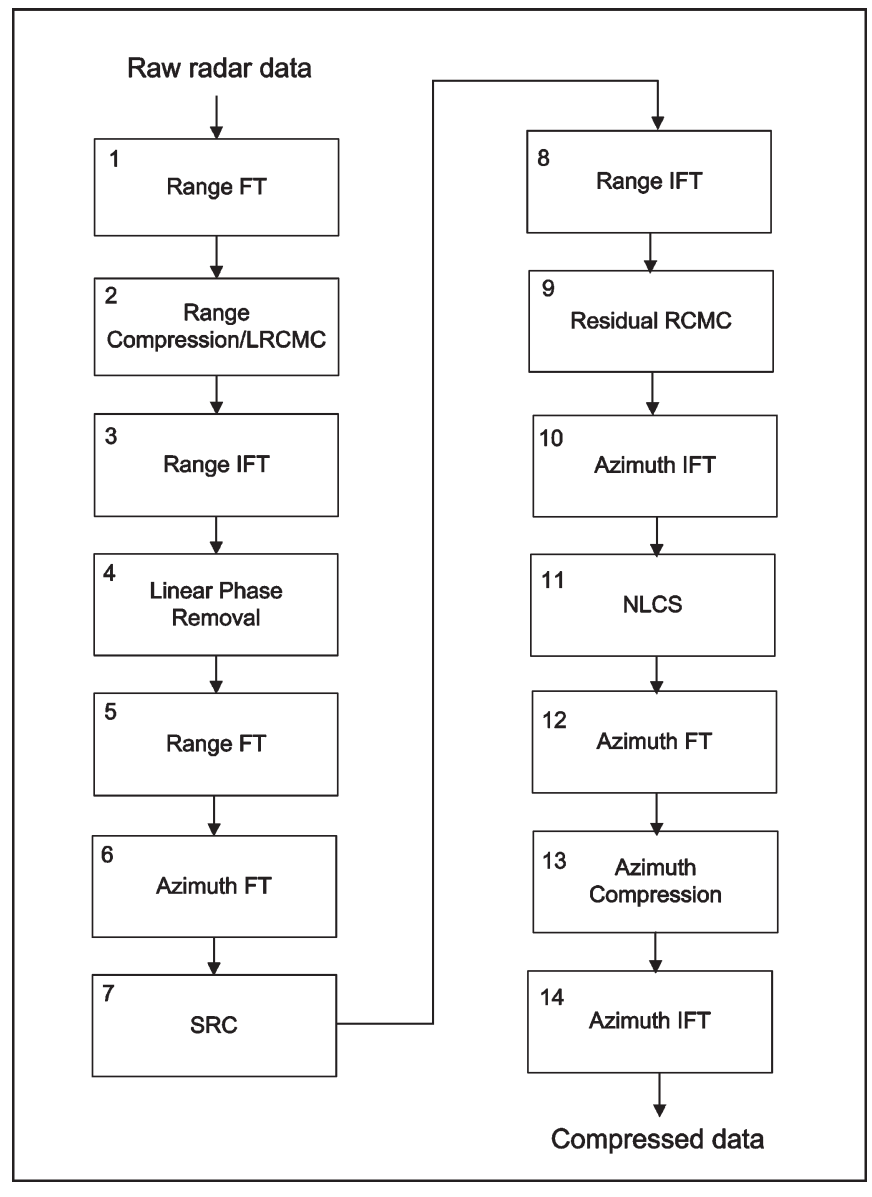

Fig. 12. Functional block diagram of the extended NLCS algorithm when SRC is incorporated.

[18]. This derivation was performed in [25], except that in this paper, the coefficient $k_{A 1}=0$ because of the removal of the linear RCM. Accordingly, the phase of the coupling term for the reference target $\mathrm{A}$ is given by

$$
\begin{aligned}
& \phi_{\operatorname{src} A}\left(f_{\tau}, f_{\eta}\right) \approx 2 \pi \frac{\lambda}{4 k_{A 2}}\left[\left(\frac{f_{\tau}}{f_{o}}\right)^{2}-\left(\frac{f_{\tau}}{f_{o}}\right)^{3}\right] f_{\eta}^{2} \\
& +2 \pi \frac{k_{A 3}}{8 k_{A 2}^{3}} \lambda^{2}\left[3\left(\frac{f_{\tau}}{f_{o}}\right)^{2}-4\left(\frac{f_{\tau}}{f_{o}}\right)^{3}\right] f_{\eta}^{3} \\
& +2 \pi \frac{9 k_{A 3}^{2}-4 k_{A 2} k_{A 4}}{64 k_{A 2}^{5}} \\
& \times \lambda^{3}\left[6\left(\frac{f_{\tau}}{f_{o}}\right)^{2}-10\left(\frac{f_{\tau}}{f_{o}}\right)^{3}\right] f_{\eta}^{4} .
\end{aligned}
$$

The coupling is azimuth frequency dependent and increases away from the zero azimuth frequency, as seen in the 1-m resolution 15-km range L-band example in Fig. 13(a). Fig. 13(b) shows how the data are less dispersed after SRC, whereas panels (c) and (d) show the considerable improvement in range resolution when SRC is applied.

\section{A. Requirement for SRC}

The extra FT and IFT operations shown in Fig. 12 require a significant amount of additional computations; thus, SRC should only be used when necessary. The phase of the residual (a)

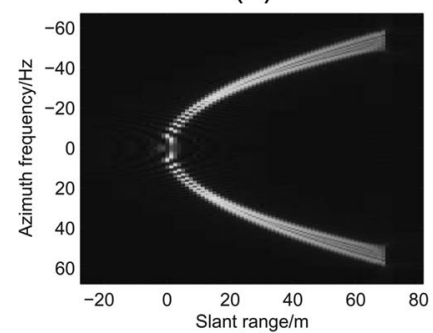

(c)

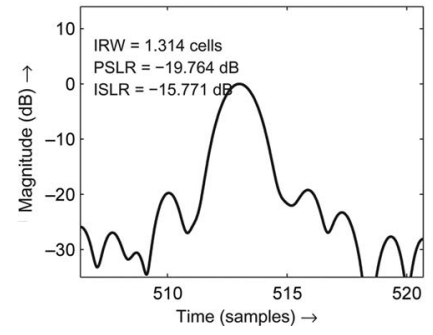

(b)

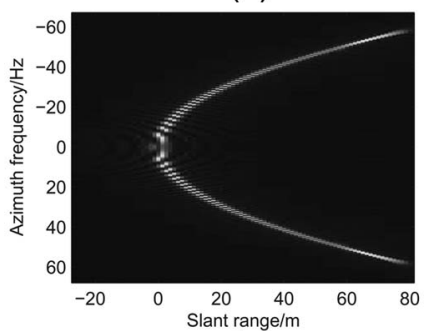

(d)

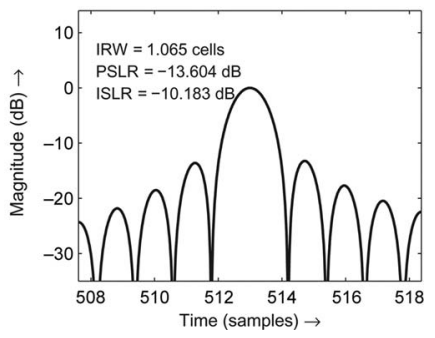

Fig. 13. Illustrating the improvement of the range resolution when SRC is added. (a) Signal in range Doppler before SRC. (b) Signal in range Doppler after SRC. (c) Range-compressed target without SRC. (d) Range-compressed target with SRC.

crosscoupling can be analyzed to determine the requirement for SRC [28]. It is sufficient to examine the dominant terms in (29). Neglecting the terms involving $f_{\tau}^{3}$ and $f_{\eta}^{4}$, as well as higher order ones, the SRC phase can be approximated by

$$
\phi_{\operatorname{src} A}\left(f_{\tau}, f_{\eta}\right) \approx \pi f_{\tau}^{2}\left[\frac{\lambda}{2 k_{A 2} f_{o}^{2}} f_{\eta}^{2}+\frac{3 \lambda^{2} k_{A 3}}{4 k_{A 2}^{3} f_{o}^{2}} f_{\eta}^{3}\right] .
$$

If SRC is not applied, the maximum phase error in the spectrum is given by (30), which is evaluated at the edges of the range and azimuth spectra. The azimuth spectral edge is at $f_{\eta}=B_{a} / 2$, and the range spectral edge is at $f_{\tau}=K_{r} T_{p} / 2$, where $B_{a}$ is the Doppler bandwidth, $T_{p}$ is the pulse length, and $K_{r}$ is the range FM rate.

If this phase error is greater than the error budget at the bandwidth extremities, SRC should be applied to the processing. Without applying the SRC filter, the coupling can result in a poor range impulse response, as shown by the example in Fig. 13(c) and (d).

\section{B. Discussion}

The operations shown in Fig. 12 represent the most rigorous way of implementing SRC in the NLCS algorithm. A number of simplifications can be examined, depending on the radar and geometry parameters, as done in [28, Sec. 6.4.1]. For example, linear phase removal may be done without the range IFT and range FT, as its dependence on range is small within the range invariance region; see (11). This saves an FT pair.

Another factor to consider is the invariance region. The invariance region tends to be quite small for highly squinted longwavelength wide-aperture cases. For instance, for a monostatic L-band $1-\mathrm{m}$-resolution system with a squint of $30^{\circ}$ at $20-\mathrm{km}$ range, the range invariance region is only $600 \mathrm{~m}$ for $5 \%$ resolution broadening. Several overlapping invariance blocks are required to cover a practical range swath of about $3 \mathrm{~km}$ or more. This will further reduce the overall efficiency of the algorithm. 


\section{APPENDIX B}

\section{DeRIVATION OF THE NLCS PERTURBATION COEFFICIENT}

This appendix derives the coefficient $\alpha$ in the perturbation function in (15). The phases of targets A and C after perturbation are written down. Subsequently, the cubic coefficient of the perturbation function is selected in such a way that the FM rates of targets $\mathrm{A}$ and $\mathrm{C}$ become equalized.

The azimuth signal for reference target $\mathrm{A}$, after introducing the cubic perturbation function $\exp \left\{j \pi \alpha \eta^{3}\right\}$, is given by

$$
s_{A \text { pert }}(\tau, \eta)=s_{A}(\tau, \eta) \exp \left\{j \pi \alpha \eta^{3}\right\}
$$

where the parameter $\alpha$ is the coefficient of the cubic perturbation function to be determined.

Then, combining (7), (10), and (12) and ignoring terms higher than the quadratic, the perturbed signal is given by

$$
\begin{aligned}
s_{\text {Apert }}(\tau, \eta) \approx & \rho_{r}(\tau- \\
\times \exp \{- & -j \frac{\pi}{\lambda}\left[\frac{V_{\mathrm{cen} A}^{2} \cos ^{2}\left(\theta_{\mathrm{sq} T}\right)}{R_{T \operatorname{cen} A}}\right. \\
& \left.\left.+\frac{V_{R}^{2} \cos ^{2}\left(\theta_{\mathrm{sq} R}\right)}{R_{R \operatorname{cen} A}}\right] \eta^{2}\right\} .
\end{aligned}
$$

Note that the constant phase term $\exp \left\{-j(2 \pi / \lambda) R_{\operatorname{cen} A}\right\}$ has been ignored here and in subsequent equations. Because the approximation in (32) only includes the second-order phase terms, only the linear FM rate component of the targets will be equalized by NLCS. At the same time, all other effects, such as the nonstationarity of the bistatic configuration, that create changes in the quadratic phase of the targets can be equalized by NLCS because this quadratic component can be absorbed into the second exponential term in (32).

Similarly, the signal of target C after LRCMC and NLCS can be written as

$$
\begin{aligned}
& s_{C \text { pert }}(\tau, \eta) \approx \rho_{r}(\tau- \\
& \times \exp \{\left.-j \frac{R_{\mathrm{cen} A}}{c}\right) w_{\mathrm{az}}\left(\eta-\eta_{C}\right) \exp \left\{j \pi \alpha \eta^{3}\right\} \\
& R_{T \operatorname{con} C}^{2}\left(\theta_{\mathrm{sq} T}\right) \\
&\left.\left.\quad+\frac{V_{R}^{2} \cos ^{2}\left(\theta_{\mathrm{sq} R}\right)}{R_{R \operatorname{cen} C}}\right]\left(\eta-\eta_{C}\right)^{2}\right\} .
\end{aligned}
$$

Note that the squint angles of target $\mathrm{C}$ are assumed to be the same as target A within the invariance region. Using target $\mathrm{A}$ as the reference, the round trip range of target $\mathrm{C}$ can be approximated as (see Fig. 3)

$$
\begin{aligned}
\Delta R_{L} & =\Delta R_{T}+\Delta R_{R} \\
& =-\left(V_{T} \sin \left(\theta_{\mathrm{sq} T}\right)+V_{R} \sin \left(\theta_{\mathrm{sq} R}\right)\right) \eta_{C} \\
R_{T \operatorname{cen} C} & =R_{T \operatorname{cen} A}+\Delta R_{T} \\
& \approx R_{T \operatorname{cen} A}-\frac{R_{T \operatorname{cen} A}}{R_{\operatorname{cen} A}}\left(V_{T} \sin \left(\theta_{\mathrm{sq} T}\right)+V_{R} \sin \left(\theta_{\mathrm{sq} R}\right)\right) \eta_{C} \\
R_{R \operatorname{cen} C} & =R_{R \operatorname{cen} A}+\Delta R_{R} \\
& \approx R_{R \operatorname{cen} A}-\frac{R_{R \operatorname{cen} A}}{R_{\operatorname{cen} A}}\left(V_{T} \sin \left(\theta_{\mathrm{sq} T}\right)+V_{R} \sin \left(\theta_{\mathrm{sq} R}\right)\right) \eta_{C}
\end{aligned}
$$

Substituting (35) and (36) into (33) gives

$$
\begin{aligned}
& s_{C \text { pert }}(\tau, \eta) \\
& \approx \rho_{r}\left(\tau-\frac{R_{\mathrm{cen} A}}{c}\right) w_{\mathrm{az}}\left(\eta-\eta_{C}\right) \exp \left\{j \pi \alpha \eta^{3}\right\} \\
& \times \exp \left\{-j \frac{\pi}{\lambda}\left[\frac{V_{T}^{2} \cos ^{2}\left(\theta_{\mathrm{sq} T}\right)}{R_{T \operatorname{cen} A}}\left(1+\frac{\Delta R_{T}}{R_{T \operatorname{cen} A}}\right)^{-1}\right.\right. \\
& \left.\left.\quad+\frac{V_{R}^{2} \cos ^{2}\left(\theta_{\mathrm{sq} R}\right)}{R_{R \operatorname{cen} A}}\left(1+\frac{\Delta R_{R}}{R_{R \operatorname{cen} A}}\right)^{-1}\right]\left(\eta-\eta_{C}\right)^{2}\right\} .
\end{aligned}
$$

Expanding the terms further, we have

$$
\begin{aligned}
& \left(1+\frac{\Delta R_{T}}{R_{\operatorname{cen} A}}\right)^{-1}=1-\frac{\Delta R_{T}}{R_{\operatorname{cen} A}}+\left(\frac{\Delta R_{T}}{R_{\operatorname{cen} A}}\right)^{2}+\cdots \\
& \left(1+\frac{\Delta R_{R}}{R_{\operatorname{cen} A}}\right)^{-1}=1-\frac{\Delta R_{R}}{R_{\operatorname{cen} A}}+\left(\frac{\Delta R_{R}}{R_{\operatorname{cen} A}}\right)^{2}+\cdots
\end{aligned}
$$

Using only the first two terms in (38) and (39) and replacing $\left(\eta-\eta_{C}\right)$ by the shifted azimuth time $\eta_{1}$, the perturbed signal at target $\mathrm{C}[(37)]$ can be written as

$$
\begin{array}{r}
s_{C \text { pert }}^{\prime}\left(\tau, \eta_{1}\right) \\
\approx \rho_{r}\left(\tau-\frac{R_{\mathrm{cen} A}}{c}\right) w_{\mathrm{az}}\left(\eta_{1}\right) \exp \left\{j \pi \alpha\left(\eta_{1}+\eta_{C}\right)^{3}\right\} \\
\times \exp \left\{-j \frac{\pi}{\lambda}\left[\frac{V_{T}^{2} \cos ^{2}\left(\theta_{\mathrm{sq} T}\right)}{R_{T \operatorname{cen} A}}\left(1-\frac{\Delta R_{T}}{R_{T \operatorname{cen} A}}\right)\right.\right. \\
\left.\left.\quad+\frac{V_{R}^{2} \cos ^{2}\left(\theta_{\mathrm{sq} R}\right)}{R_{R \operatorname{cen} A}}\left(1-\frac{\Delta R_{R}}{R_{R \operatorname{cen} A}}\right)\right] \eta_{1}^{2}\right\}
\end{array}
$$

where $s^{\prime}($.$) is a version of s($.$) , which is shifted by \eta_{C}$. Expanding (40), the phase terms can be collected as

$$
\begin{aligned}
& s_{C \text { pert }}^{\prime}\left(\tau, \eta_{1}\right) \\
& \approx \rho_{r}\left(\tau-\frac{R_{\mathrm{cen} A}}{c}\right) w_{\mathrm{az}}\left(\eta_{1}\right) \\
& \times \exp \left\{j \pi \alpha\left(\eta_{1}^{3}+3 \eta_{C} \eta_{1}^{2}+3 \eta_{C}^{2} \eta_{1}+\eta_{C}^{3}\right)\right\} \\
& \quad \times \exp \left\{-j \frac{\pi}{\lambda}\left(\frac{V_{T}^{2} \cos ^{2}\left(\theta_{\mathrm{sq} T}\right)}{R_{T \operatorname{cen} A}}+\frac{V_{R}^{2} \cos ^{2}\left(\theta_{\mathrm{sq} R}\right)}{R_{R \operatorname{cen} A}}\right) \eta_{1}^{2}\right\} \\
& \quad \times \exp \left\{-j \frac{\pi}{\lambda}\left(\frac{V_{T}^{2} \cos ^{2}\left(\theta_{\mathrm{sq} T}\right)}{R_{T \operatorname{cen} A} R_{\mathrm{cen} A}}+\frac{V_{R}^{2} \cos ^{2}\left(\theta_{\mathrm{sq} R}\right)}{R_{R \operatorname{cen} A} R_{\mathrm{cen} A}}\right)\right. \\
& \left.\quad \times\left(V_{T} \sin \left(\theta_{\mathrm{sq} T}\right)+V_{R} \sin \left(\theta_{\mathrm{sq} R}\right)\right) \eta_{C} \eta_{1}^{2}\right\} .
\end{aligned}
$$

To find the coefficient $\alpha$, the sum of the quadratic terms in $\eta_{1}$ that involve the target position parameter $\eta_{C}$ is set to zero, and the result is given in (16). The remaining terms in the perturbed signal are given in (17).

\section{APPENDIX C \\ INVARIANCE REGION ANALYSIS}

This appendix shows how the invariance region size can be determined, and it depends on the imaging geometry and radar parameters for each case. The size of the invariance region is limited by a number of factors. Often, the governing factors are the residual RCM and quadratic phase errors (QPE), and the discussion that follows is limited to these two factors only. 
Let the ranges and squint angles of the transmitter and receiver to a target at position $\left(\delta_{x}, \delta_{y}\right)$ be defined by $R_{T}\left(\delta_{x}, \delta_{y}\right), R_{R}\left(\delta_{x}, \delta_{y}\right), \theta_{T}\left(\delta_{x}, \delta_{y}\right)$, and $\theta_{R}\left(\delta_{x}, \delta_{y}\right)$, where $\delta_{x}$ and $\delta_{y}$ are the range and azimuth offsets, respectively, from the reference target $\mathrm{A}$ in the coordinate system after LRCMC. Note that the position of target $\mathrm{A}$ is $(0,0)$ and these four parameters are given for target $\mathrm{A}$ as $R_{T \operatorname{cen} A}, R_{R \operatorname{cen} A}, \theta_{\mathrm{sq} T}$, and $\theta_{\mathrm{sq} R}$ in the main text.

Processing is done by keeping the squint angles within a processing block constant. Using (8), the residual linear RCM of a target for an exposure time $T_{a}$ is given by

$$
\begin{array}{r}
\Delta R_{\mathrm{lrcm}}\left(\delta_{x}, \delta_{y}\right)=\left[V_{T} \sin \left(\theta_{T}\left(\delta_{x}, \delta_{y}\right)\right)+V_{R} \sin \left(\theta_{R}\left(\delta_{x}, \delta_{y}\right)\right)\right] T_{a} \\
-\left[V_{T} \sin \left(\theta_{T}(0,0)\right)+V_{R} \sin \left(\theta_{R}(0,0)\right)\right] T_{a} .
\end{array}
$$

Similarly, it can be deduced from (17) that the quadratic RCM of the target is

$$
\begin{array}{r}
\Delta R_{\mathrm{qrcm}}\left(\delta_{x}, \delta_{y}\right)=\frac{1}{2}\left\{\frac{V_{T}^{2} \cos ^{2}\left(\theta_{T}\left(\delta_{x}, \delta_{y}\right)\right)}{R_{T}\left(\delta_{x}, \delta_{y}\right)}\right. \\
\left.+\frac{V_{R}^{2} \cos ^{2}\left(\theta_{R}\left(\delta_{x}, \delta_{y}\right)\right)}{R_{R}\left(\delta_{x}, \delta_{y}\right)}\right\}\left(\frac{T_{a}}{2}\right)^{2} \\
-\frac{1}{2}\left\{\frac{V_{T}^{2} \cos ^{2}\left(\theta_{T}(0,0)\right)}{R_{T}\left(\delta_{x}, \delta_{y}\right)}\right. \\
\left.+\frac{V_{R}^{2} \cos ^{2}\left(\theta_{R}(0,0)\right)}{R_{R}\left(\delta_{x}, \delta_{y}\right)}\right\}\left(\frac{T_{a}}{2}\right)^{2} .
\end{array}
$$

Because RCMC is corrected to target A, the residual RCM of the target at $\left(\delta_{x}, \delta_{y}\right)$ is the sum of the residual linear and quadratic components, and it is given by

$$
\Delta R_{\mathrm{rcm}}\left(\delta_{x}, \delta_{y}\right)=\left|\Delta R_{\mathrm{lrcm}}\left(\delta_{x}, \delta_{y}\right)\right|+\left|R_{\mathrm{qrcm}}\left(\delta_{x}, \delta_{y}\right)\right| .
$$

Within a processing block, the residual RCM should be kept within a fraction of one range resolution cell $\rho_{r}$. Let this fraction be $\gamma_{\mathrm{rcm}}$, which is nominally set to be less than 1 . The maximum range deviation $\Delta x_{\mathrm{rcm}}$ from target $\mathrm{A}$ is found from the following:

$$
\Delta R_{\mathrm{rcm}}\left(\Delta x_{\mathrm{rcm}}, 0\right)<\gamma_{\mathrm{rcm}} \rho_{r} .
$$

Similarly, the maximum azimuth deviation $\Delta y_{\mathrm{rcm}}$ from target $\mathrm{A}$ is found from the following:

$$
\Delta R_{\mathrm{rcm}}\left(0, \Delta y_{\mathrm{rcm}}\right)<\gamma_{\mathrm{rcm}} \rho_{r}
$$

Moreover, within a processing block, the QPE should be limited by $\gamma_{\mathrm{qpe}} \pi$, where $\gamma_{\mathrm{qpe}}$ is nominally set to less than 0.5 . As the QPE is governed by the residual quadratic RCM, the maximum range deviation $\Delta x_{\mathrm{qpe}}$ from target $\mathrm{A}$ is found from the condition

$$
\frac{2 \pi}{\lambda}\left|\Delta R_{\mathrm{qrcm}}\left(\Delta x_{\mathrm{qpe}}, 0\right)\right|<\gamma_{\mathrm{qpe}}
$$

and the maximum azimuth deviation $\Delta y_{\mathrm{qpe}}$ is found from the following:

$$
\frac{2 \pi}{\lambda}\left|\Delta R_{\mathrm{qrcm}}\left(0, \Delta y_{\mathrm{qpe}}\right)\right|<\gamma_{\mathrm{qpe}} \pi .
$$

Finally, the range invariance region size is as follows:

$$
\Delta X_{\mathrm{ir}}=2 \min \left\{\Delta x_{\mathrm{rcm}}, \Delta x_{\mathrm{qpe}}\right\}
$$

and the azimuth invariance region size is as follows:

$$
\Delta Y_{\text {ir }}=2 \min \left\{\Delta y_{\text {rcm }}, \Delta y_{\mathrm{qpe}}\right\} .
$$

\section{ACKNOWLEDGMENT}

The authors would like to thank Prof. O. Loffeld of the Zentrum für Sensorsysteme, University of Siegen, for hosting Dr. Neo during the last three months of his Ph.D. program. The authors also like to thank the reviewers who made detailed constructive comments that allowed us to considerably improve the manuscript.

\section{REFERENCES}

[1] D. D'Aria, A. M. Guarnieri, and F. Rocca, "Focusing bistatic synthetic aperture radar using dip move out," IEEE Trans. Geosci. Remote Sens., vol. 42, no. 7, pp. 1362-1376, Jul. 2004.

[2] I. Walterscheid, J. H. G. Ender, A. R. Brenner, and O. Loffeld, "Bistatic SAR processing using an $\omega-k$ type algorithm," in Proc. IGARSS, Seoul, Korea, Aug. 2005, pp. 1064-1067.

[3] V. Giroux, H. Cantalloube, and F. Daout, "Frequency domain algorithm for bistatic SAR," in Proc. EUSAR, Dresden, Germany, May 2006. CD-ROM.

[4] Z. Zhang, M. Xing, J. Ding, and Z. Bao, "Focusing parallel bistatic SAR data using the analytic transfer function in the wavenumber domain," IEEE Trans. Geosci. Remote Sens., vol. 45, no. 11, pp. 3633-3645, Nov. 2007.

[5] R. Bamler and E. Boerner, "On the use of numerically computed transfer function for processing of data from bistatic SARs and high squint orbital SARs," in Proc. IGARSS, Seoul, Korea, Jul. 2005, vol. 2, pp. 1051-1055.

[6] R. Bamler, F. Meyer, and W. Liebhart, "Processing of bistatic SAR data from quasi-stationary configurations," IEEE Trans. Geosci. Remote Sens., vol. 45, no. 11, pp. 3350-3358, Nov. 2007.

[7] D. Hale, "Dip-moveout by Fourier transform," Geophysics, vol. 49, no. 14 , pp. 741-757, Jun. 1984.

[8] A. Monti Guarnieri and F. Rocca, "Reduction to monostatic focusing of bistatic or motion uncompensated SAR surveys," Proc. Inst. Electr. Eng.-Radar, Sonar Navig., vol. 153, no. 3, pp. 254-261, Jun. 2006.

[9] O. Loffeld, H. Nies, V. Peters, and S. Knedlik, "Models and useful relations for bistatic SAR processing," IEEE Trans. Geosci. Remote Sens., vol. 42, no. 10, pp. 2031-2038, Oct. 2004.

[10] K. Natroshvili, O. Loffeld, H. Nies, and A. M. Ortiz, "First steps to bistatic focusing," in Proc. IGARSS, Seoul, Korea, Jul. 2003, pp. 1072-1076.

[11] K. Natroshvili, O. Loffeld, and H. Nies, "Focusing of arbitrary bistatic SAR configurations," in Proc. EUSAR, Dresden, Germany, May 2006. CD-ROM.

[12] K. Natroshvili, O. Loffeld, H. Nies, A. M. Ortiz, and S. Knedlik, "Focusing of general bistatic SAR configuration data with 2-D inverse scaled FFT," IEEE Trans. Geosci. Remote Sens., vol. 44, no. 10, pp. 2718-2727, Oct. 2006.

[13] M. Rodríguez-Cassolá, G. Krieger, and M. Wendler, "Azimuth-invariant, bistatic airborne SAR processing strategies based on monostatic algorithms," in Proc. IGARSS, Seoul, Korea, Aug. 2005, pp. 1047-1050.

[14] C. Wu, K. Y. Liu, and M. J. Jin, "A modeling and correlation algorithm for spaceborne SAR signals," IEEE Trans. Aerosp. Electron. Syst., vol. AES18, no. 5, pp. 563-574, Sep. 1982.

[15] R. K. Raney, H. Runge, R. Bamler, I. G. Cumming, and F. H. Wong, "Precision SAR processing using chirp scaling," IEEE Trans. Geosci. Remote Sens., vol. 32, no. 4, pp. 786-799, Jul. 1994.

[16] F. H. Wong and T. S. Yeo, "New applications of non-linear chirp scaling in SAR data processing," IEEE Trans. Geosci. Remote Sens., vol. 39, no. 5, pp. 946-953, May 2001. 
[17] Q. Xiaolan, H. Donghui, and D. Chibiao, "Non-linear chirp scaling algorithm for one-stationary bistatic SAR," in Proc. 1st Asian Pacific Conf. Synthetic Aperture Radar, Huangshan, China, Nov. 5-9, 2007, pp. 111-114.

[18] Y. L. Neo, F. H. Wong, and I. G. Cumming, "A two-dimensional spectrum for bistatic SAR processing using series reversion," IEEE Geosci. Remote Sens. Lett., vol. 4, no. 1, pp. 93-96, Jan. 2007.

[19] D. C. Munson, J. D. O'Brian, and W. K. Jenkins, "A tomographic formulation of spotlight mode synthetic aperture radar," Proc. IEEE, vol. 71, no. 8, pp. 917-925, Aug. 1983.

[20] Y. L. Neo, "Digital processing algorithms for bistatic synthetic aperture radar data," Ph.D. dissertation, Dept. Electr. Comput. Eng., Univ. British Columbia, Vancouver, BC, Canada, May 2007.

[21] A. Moreira, J. Mittermayer, and R. Scheiber, "Extended chirp scaling algorithm for air and spaceborne SAR data processing in stripmap and ScanSAR imaging modes," IEEE Trans. Geosci. Remote Sens., vol. 34, no. 5, pp. 1123-1136, Sep. 1996.

[22] Y. L. Neo, I. G. Cumming, and F. H. Wong, "Bistatic SAR processing using non-linear chirp scaling," in Proc. CEOS SAR Calibration Workshop (ESA), Ulm, Germany, May 27-28, 2004. CD-ROM.

[23] Y. L. Neo, F. H. Wong, and I. G. Cumming, "Focusing bistatic SAR images using non-linear chirp scaling," in Proc. IEEE/URSI Int. Conf. Radar Syst. (RADAR), Toulouse, France, Oct. 18-22, 2004.

[24] Y. Neo, F. H. Wong, and I. G. Cumming, "An efficient non-linear chirp scaling method of focusing bistatic SAR images," in Proc. EUSAR, Dresden, Germany, May 2006. CD-ROM.

[25] Y. L. Neo, F. H. Wong, and I. G. Cumming, "Processing of azimuthinvariant bistatic SAR data using the range Doppler algorithm," IEEE Trans. Geosci. Remote Sens., vol. 46, no. 1, pp. 14-21, Jan. 2008.

[26] G. Krieger, H. Fiedler, D. Houman, and A. Moreira, "Analysis of system concepts for bi- and multi-static SAR missions," in Proc. IGARSS, Toulouse, France, Jul. 2003, pp. 770-772.

[27] I. Walterscheid, A. R. Brenner, and J. H. G. Ender, "Results on bistatic synthetic aperture radar," Electron. Lett., vol. 40, no. 19, pp. 1224-1225, Sep. 2004.

[28] I. G. Cumming and F. H. Wong, Digital Processing of Synthetic Aperture Radar Data: Algorithms and Implementation. Norwood, MA: Artech House, 2005.

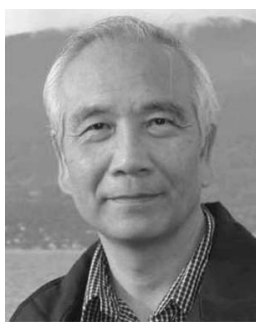

Frank H. Wong received the B.Eng. degree in electrical engineering from McGill University, Montreal, QC, Canada, the M.Sc. degree in electrical engineering from Queen's University, Kingston, ON, Canada, and the Ph.D. degree in computer science from the University of British Columbia (UBC), Vancouver, BC, Canada.

Since 1977, he has been with MacDonald, Dettwiler and Associates Ltd., Richmond, BC, where he worked in the Landsat and SPOT imaging area for the first few years. Then, his interest switched to synthetic aperture radar, and he has been working on airborne and satellite SAR processing and Doppler estimation ever since. He spent a sabbatical in the Radar Remote Sensing Group of the Department of Electrical and Computer Engineering, UBC, in 1994. From 1986 to 2004, he was a Sessional Lecturer on image processing with the Department of Computer Science, UBC. He was a Visitor with the National University of Singapore, Singapore, in 1999, where he did research in bistatic SAR processing. He is a coauthor of Digital Processing of Synthetic Aperture Data-Algorithms and Implementation (Artech House, 2005).

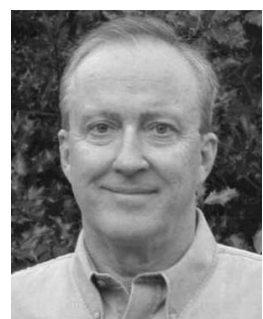

Ian G. Cumming (S'63-M'66-SM'05-LSM'06) received the B.Sc. degree in engineering physics from the University of Toronto, Toronto, ON, Canada, in 1961 and the Ph.D. degree in computing and automation from the Imperial College, University of London, London, U.K., in 1968.

In 1977, he was with MacDonald, Dettwiler and Associates Ltd., Richmond, BC, Canada, where he developed synthetic aperture radar (SAR) signal processing algorithms, including Doppler estimation and autofocus routines. He has been involved in the algorithm design of digital SAR processors for SEASAT, SIR-B, ERS-1/2, J-ERS-1, and RADARSAT, as well as several airborne radar systems. He was a Visiting Scientist with the German Aerospace Center, Oberpfaffenhofen, in 1999. Since 1993, he has been with the Department of Electrical and Computer Engineering, University of British Columbia, Vancouver, BC, where he held the MacDonald Dettwiler/NSERC Industrial Research Chair in radar remote sensing from 1993 to 2003. The Radar Remote Sensing Group has published papers in the fields of SAR processing, SAR data encoding, satellite SAR two-pass interferometry, airborne along-track interferometry, polarimetric radar image classification, and SAR Doppler estimation. He is a coauthor of Digital Processing of Synthetic Aperture Data-Algorithms and Implementation (Artech House, 2005). Since 2006, he has been a Professor Emeritus with the department.

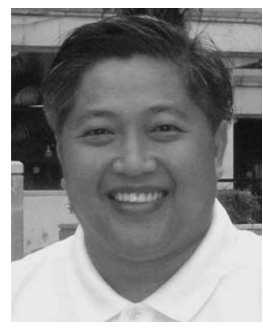

Yew Lam Neo (S'06-M'07) received the B.Eng. degree in electrical engineering from the National University of Singapore, Singapore, in 1994 and the $\mathrm{Ph} . \mathrm{D}$. degree in electrical engineering from the University of British Columbia, Vancouver, BC, Canada, in 2007 .

From 1994 to 2001, he was an Engineer with DSO National Laboratories, Singapore, where he worked on SAR systems and software for real-time embedded systems. In 2006, he was a Visiting Scientist for three months with the Zentrum für Sensorsysteme, University of Siegen, Siegen, Germany. He is currently with the Sensor Division, Radar Techniques Laboratory, DSO National Laboratories, where he is continuing his research in bistatic SAR signal processing.

Dr. Neo was awarded the Engineering Book Prize and the winner of the Innovation Prize Award for his final year project work on a very high frequency synthesizer. 